\title{
Automatic Initialization of Contour for Level Set Algorithms Guided by Integration of Multiple Views to Segment Abdominal CT Scans
}

\author{
Mahmoud Saleh Jawarneh ${ }^{1}$, Rajeswari Mandava ${ }^{1}$, Dhanesh Ramachandram ${ }^{1}$, Ibrahim Lutfi Shuaib ${ }^{2}$. \\ ${ }^{1}$ School of Computer Sciences, ${ }^{2}$ Advanced Medical \& Dental Institute \\ Universiti Sains Malaysia, \\ ${ }^{1} 11800$ Minden, ${ }^{2} 13200$ Kepala Batas, Penang, Malaysia \\ \{jawarneh, mandava, dhaneshr\}@cs.usm.my,ibrahim@amdi.usm.edu.my
}

\begin{abstract}
This paper presents a new automatic initialization procedure for a level-set based segmentation algorithm that works on all slices for a given CT dataset. Level set segmentation algorithms provide promising results, are robust to dataset variations and do not require prior training. As such, they can be reliably used for segmentation of major organs in abdominal CT scans. However, level set algorithms still require user intervention to plot the initial contour for each slice in a given dataset, which is a time consuming process. Therefore, we propose here, a technique of using multiple views to automatically initialize and propagate the contour through each slice in the $\mathrm{CT}$ dataset. The technique requires a user to only initialize a single point within the organ of interest in order to initiate the automated segmentation process. We report the segmentation results for liver and spleen organs within the abdominal region using three different datasets. We conclude that this technique can be used to reduce the processing time for any level set algorithm suitable to abdominal CT scans. We typically achieve time efficiency up to $203.03 \%$ for complete segmentation of three organs as compared to manually initializing the level set contour for each slice.
\end{abstract}

Keywords-automatic initialization; mutliple views integration; level set active contour; abdominal CT images; medical knowledge.

\section{INTRODUCTION}

Segmentation of abdominal organs presents several challenges in CT images, showing high similarities in the gray levels among different organs and the surrounding soft tissues and inhomogeneity in shape and texture of organ tissues within and among different image slices. Additional difficulties are encountered when dealing with CT scans that have low contrast and blurred edges, due to partial volume effects resulting from spatial averaging, patient movement, beam hardening and reconstruction artifacts, as well as heartbeat and breathing [1].

The need for automatic segmentation of abdominal CT scans is ever growing. Currently, the segmentation is performed by experts who delineate organs borders on each slice in the volume dataset with the aid of semi-automatic tools. The result of segmentation in these processes depends on the skills of the operator in dealing with these tools and his/her knowledge of the target organ which suffer from his/her errors and biases, and also because these methods are tedious and time consuming [2].

There are several abdominal organ segmentation methods in CT images; including neural network learning techniques which most of these methods strongly depend on and require serious training set to build the shape and statistical or contextual constraints of organs to feed into neural network [3] [4]. Gray level based techniques such as thresholding, edge detection; mathematical morphology and region growing are required of the initial values, to start the segmentation and are based on intensity similarity. Over segmentation occurs when adjacent tissues have similar intensity to the target organ [2]. Rule-based recognition [3] on the other hand, is based on exploiting organ invariants and features such as size, location, edges and gray levels. Model-based techniques need training sets to be manually segmented and properly collected to produce the model correctly placed in dataset to give good results [2]. Atlasbased segmentation [5] [6] requires registration between built atlas and the target images. Active contour level set methods [7] [8] [9] give promising results, robust to dataset variations and not dependent on the training set. However they still require manual interaction from the user, to plot the initial contour inside specific organ. In addition, more computation is required for the level set algorithms to reach the desirable borders if the initial contour initialized farther from its final position. The focus of this paper is on the level set based active contour segmentation algorithms. And how to solve the problem of initializing the initial contour curve inside the target abdominal organ in each slice, near from the right organ's border in CT abdominal dataset by using prior knowledge to help in automatic segmentation and to reduce the segmentation time.

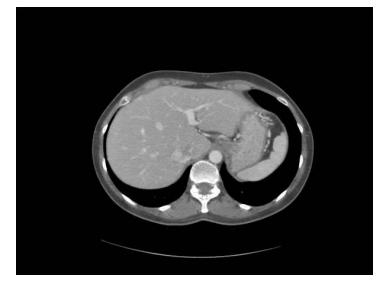

(a)

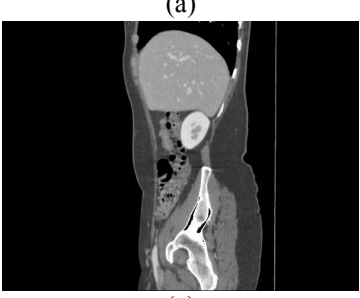

(c)

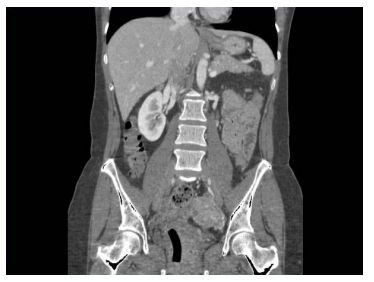

(b)

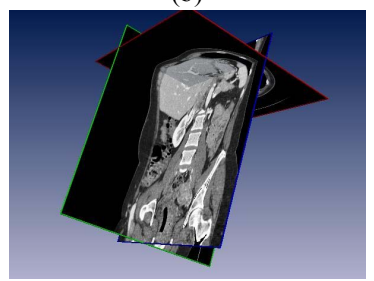

(d)
Figure 1. (a) Axial; (b) Coronal; (c) Sagittal; (d) All; views of abdominal dataset. 
The use of multiple views to guide the segmentation of human organs is common in different researches. Ray casting technique is a visualization and segmentation method used in volume rendering introduced [13]. The ray casting technique has function to calculate (average, maximum, minimum, etc depend on the ray casting function) for the intensity values on the same line (horizontal or vertical, depend on the view). It could be used to visualize (segment) some anatomies such bone and skin, depending on the ray cast function. Different views (sagittal and coronal) could also be used [14] to landmark the beginning and the end of abdominal organs manually before the segmentation. Active contour segmentation is however performed on three different planes (Axial, Sagittal and Coronal) to capture the image (brain images) [15], then a majority voting procedure is applied to classify each voxel in the image as either a white matter or not. Automatic initialization of active contour [12] has also been introduced to segment three different views axial, sagittal or coronal with no cooperation between them to strip the skull and capture brain tissues in MRI images. The propagation of contour curve from slice to next slice is based on metadata information which is here slice spacing to shrink the contour result from previous slice. This method is suitable for brain tissue, due to the large similarity in the shape of brain tissues from one slice to another, allowing the approach to propagate the contour based on metadata information only [12].

This paper describes a technique using a cooperation of multiple views of abdominal CT datasets as a prior knowledge to solve the problem of manual initializing of contour curve inside target organ in each axial slice in the abdominal CT dataset. The contour curve is manually initialized on a selected axial slice in the dataset and propagated automatically to subsequent axial slices using collaborative information available in coronal view which is able to examine if the organ increasing or decreasing in size. This information is useful to expand or shrink contour curve in the subsequent axial slice near to organ border before performing the segmentation Fig. $1 \mathrm{a}, \mathrm{b}$ and $\mathrm{c}$ shows multiple views for one of the abdominal dataset. Our research findings could thus contribute to solving the difficult and challenging problem of segmenting abdominal organs CT images.

The rest of the paper is organized as follows. In Section 2 , we give an overview of level set based active contour algorithms. Section 3, describes the proposed technique for automatic contour initialization using multiple views. In Section 4, experimental results on abdominal CT images are presented and discussed. We provide our conclusions in Section 5.

\section{LEVEL SET BASED ACTIVE CONTOUR}

The early idea on level set based active contour was introduced by Osher \& James [16]. It was introduced to overcome the limitations of the snake model. Different types of level set methods have been proposed, which could be categorized into three; edge based model, region based model and hybrid methods.
The first category; edge based model is suitable for images which have clear boundaries. It employs image gradient information as forces to stop the contour at the boundary of interest, as well as regularizing forces to minimize the length of contour and produce a smooth boundary. A general model for image segmentation given by Malladi [17] is as shown in is defined as in (1)

$$
\mathrm{F}=\mathrm{g}_{1}\left(\mathrm{~F}_{0}-\varepsilon \mathrm{k}\right)
$$

where $\mathrm{g}$ is gradient force and $\mathrm{k}$ is the regularizing force.

The second category is region based model, which utilizes region properties such as intensity, texture or color to halt the evolution of contours at desired boundaries as one force and the regularizing term to smoothen the contour as a second force. Chan \& Vese [18] introduced the active contour without edges which integrated the Mumford-Shah segmentation function with level set, using energy minimization approach as given by (2).

$$
\mathrm{E}=\int_{\omega}(\mathrm{I}-\mathrm{u})^{2} \mathrm{dA}+\int_{\bar{\omega}}(\mathrm{I}-\mathrm{v})^{2} \mathrm{dA}
$$

where; $u$ and $v$ represent the mean image intensities over $\omega$ inside and outside respectively.

The third category is the hybrid model, which integrates image gradient, region properties and prior knowledge of the image such as location and shape, to evolve the contour to suit the difficulties of segmenting abdominal images.

Several methods have been proposed and tested using level set segmentation to segment abdominal images. Transformation of closed contours of the anatomies such as human body, liver, stomach and spleens are manually segmented from atlas slice to each slice in the dataset through registration, before performing level set segmentation, as proposed in [5]. This process slows the whole operation. Pan et al [7] introduced an accumulative level set speed function which varied with time to improve the detection sensitivity of weak edges in segmenting liver incorporation with prior liver location, based on anatomy knowledge. However Pan's level set algorithm is a time consuming process because it requires the plotting of a small circle inside the liver region for each slice in the dataset manually.

Predicting the initial liver shape using 2.5D shape propagation is used to advance the level set instead of plotting curve inside each slice [8]. This method still requires a manual selection of seed points at the topmost and bottommost slice. Stop level set without edges method was developed automatically to segment liver organ based on Chan-Vese method. This method requires no a priori shape or size information but still needs manual initialization of cure in each slice in the dataset by drawing rectangular window [9]. All these approaches discussed require user interaction, to plot the contour of level set in each slice in the abdominal dataset. 


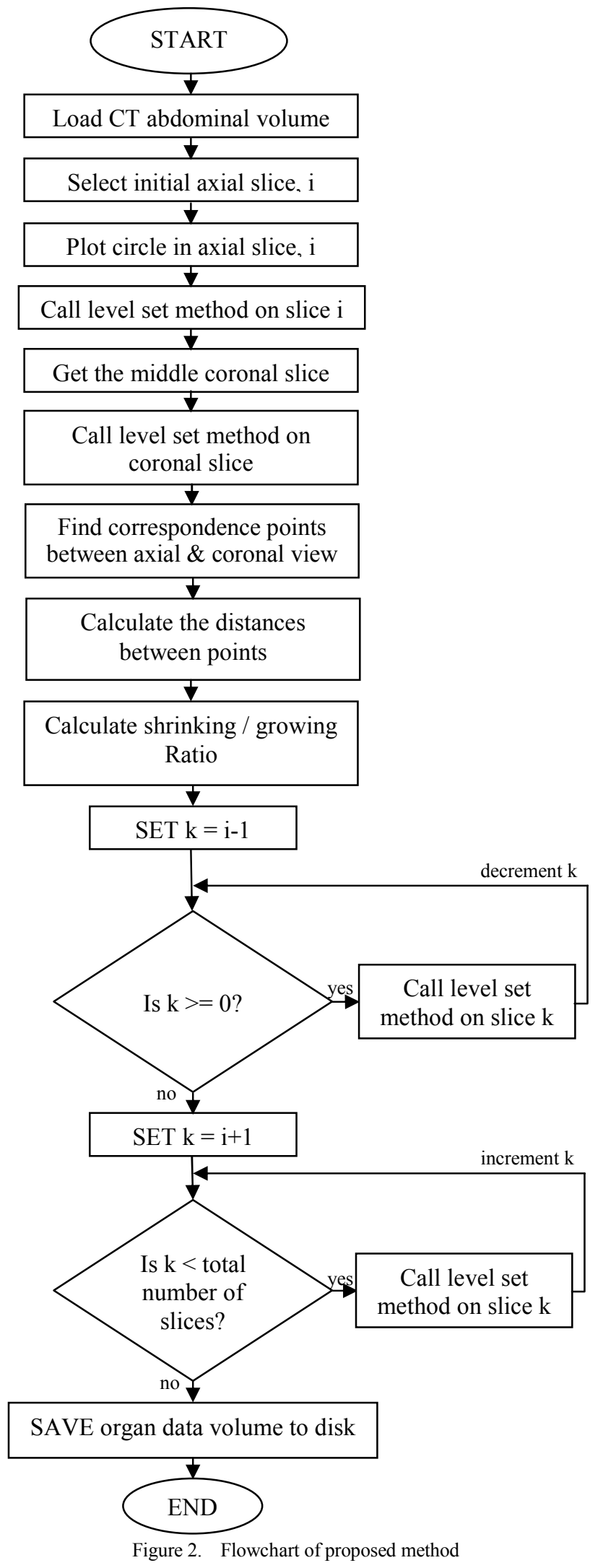

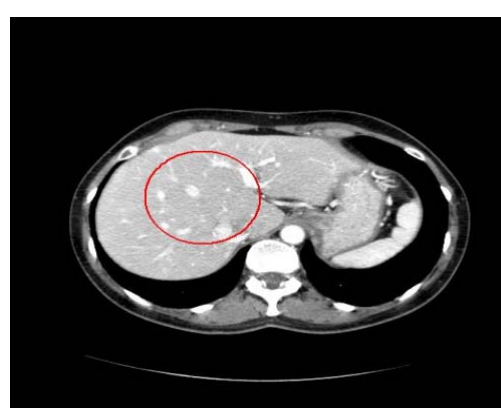

Figure 3. Initial contour inside liver.

\section{PROPOSED METHOD}

As detailed in the following sub-sections, our technique consists of three main stages: 1- Initialization of contour curve inside target organ in a chosen axial slice and segmenting the organ. 2- Segmenting the middle coronal view in the target organ. 3- Segmentation of subsequent axial slices. Fig. 2 shows an overall flowchart diagram of the method used.

\section{A. Initialization}

Initialization of the contour curve in each slice in abdominal dataset is through the use of image registration introduced by Ding [5]. It is potentially accurate but is time consuming mainly in large scale analyses. Therefore, in our approach avoid registration process and initialize the contour curve in the target organ completely within the organ area. In this stage the user is required to do nothing other to than select the initial point inside the target organ in of the axial slices. This limitation of user interaction at this step is ideal for the medical domain. The point chosen is used as the center of contour circle curve, with small radius inside the target organ in a chosen axial slice as shown in Fig. 3.

Suitable level set segmentation method could be used to segment abdominal organs such as in the Chan-Vese method, Pan, Lee and Garamendi methods. We utilize the level set introduced by Chunming et al. [19], to segment the target organ in the chosen axial slice. This approach is more efficient than the traditional level-set methods [20] since it does not require re-initialization of the level set function at every iteration. In addition, it is robust against noise and has the ability to perform well in the presence of weak boundaries.

$$
\frac{\partial \phi}{\partial \mathrm{t}}=\mu\left[\Delta \emptyset-\operatorname{div}\left(\frac{\nabla \emptyset}{|\nabla \varnothing|}\right)\right]+\lambda \delta(\varnothing) \operatorname{div}\left(\mathrm{g} \frac{\nabla \emptyset}{|\nabla \emptyset|}\right)+\operatorname{vg} \delta(\varnothing) .
$$

The variational formulation consist of internal energy represented by the first term on the right hand side of (3) to penalize the deviation of the level set function from a signed distance function, and an external energy represented by the second and the third terms in (3) correspond to the gradient flow, to drive the motion of level set curve towards the object boundaries. Fig. 4 shows the result of segmenting the liver using Chunming level set method. 


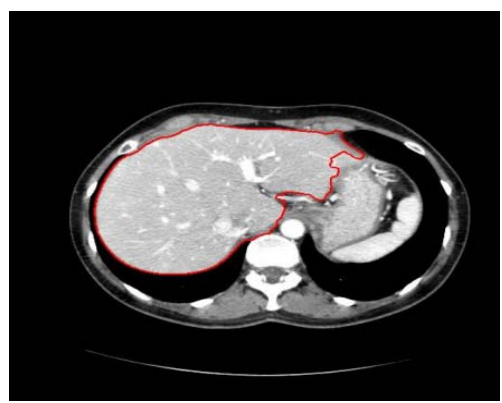

Figure 4. Level set Segmented liver.

\section{B. Segmenting coronal view}

The representation of segmented result (organ boundary) in the selected axial slice Fig. 4 is a set of boundary points $(\mathrm{x}, \mathrm{y})$, which gives the number of coronal view slices inside the organ by calculating the differences between the maximum and minimum points in $\mathrm{X}$ direction then divides by two as shown in (4), to get the middle coronal slice inside the segmented organ which capture the complete organ size.

$$
\mathrm{MC}=\frac{\operatorname{Max}(\mathrm{x})-\operatorname{Min}(\mathrm{x})}{2} \text {. }
$$

where $\mathrm{MC}$ is a middle coronal slice and $\mathrm{x}$ is $\mathrm{X}$ coordinates of segment organ boundary.

After getting the middle coronal slice again, the level set in Chunming method is applied to segment the coronal view of the target organ. Segmenting the coronal view of the organ helps to know the starting and ending axial slice of the target organ, which is useful to reduce the number of slices which are processed. For example, if abdominal dataset consists of 170 slices (as shown in Fig. 5) and needs a large memory to process and the target organ ( in this case the liver) exists in 130 slices, referring to the segmentation result of coronal view could reduce the number of the processed slices. Segmenting the coronal view also helps to ensure that the entire target organ is captured early before propagation. However, this normally requires users with sufficient anatomical knowledge to insert several points which specify the beginning and end of the target organ. The main benefit of segmenting the coronal view is to use it as prior knowledge to guide the initialization of the level set method in the subsequent axial slice, should the contour curve shrink or grow.

\section{Segmentation of subsequent slices}

Segmentation of subsequent axial slices is possible, given the continuity of the target organ surface. This is because the input datasets are a series of subsequent slices, which are closely related to each other and the organ boundary in a slice is slightly different from that in its adjacent slice. This allows the use of the resultant contour curve (segmented boundaries) from the current slice to initialize the contour in adjacent slice. This can save computation time and improve the accuracy of results. In order to guide the initialization of contour in subsequent slices, level-set active contour is performed to segment the middle coronal slice in target organ. Then the corresponding points between the boundary of the segmented organ in coronal view and all axial slices crossing these boundaries are found, as shown in Fig. 6 .

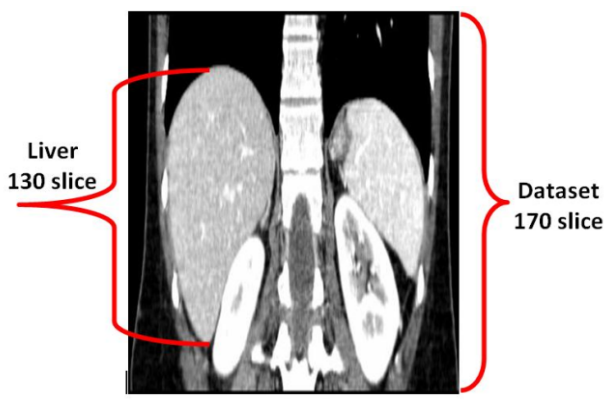

Figure 5. Abdominal dataset coronal view.

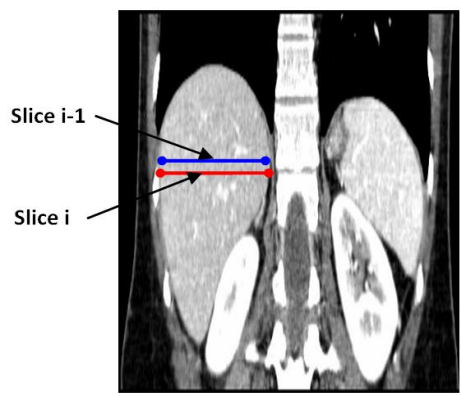

Figure 6. Correspondence points between coronal and axial views.

Afterwards, the distances between each two correspondence points in same axial slice are calculated, based on (5).

$$
\mathrm{d}=\sqrt{\left(x_{n}-x_{m}\right)^{2}+\left(y_{n}-y_{m}\right)^{2}}
$$

where $\mathrm{d}$ is the distance between two points $\left(x_{n}, y_{n}\right)$ and $\left(x_{m}, y_{m}\right)$.

The contour curve on the current axial slice is shrunk or grown before propagating the initial contour curve to the next/previous slice based on the ratio of distances between sequential axial slices as in (6).

$$
\text { Ratio }_{\mathrm{sg}}=\frac{\mathrm{d}\left(\text { Slice }_{\mathrm{i}+1 / \mathrm{i}-1}\right)}{\mathrm{d}\left(\text { Slice }_{\mathrm{i}}\right)}
$$

where, Ratio sg $_{\text {is }}$ is the ratio of contour curve shrinking or growing.

The new contour curve points calculated as in (7).

$$
\text { New contour }(x, y)=\text { Current contour }(x, y) * \text { Ratio }_{\text {sg }} \text {. }
$$

However, to keep the propagated (new) contour curves inside the organ region, the calculated contour curve is shrunk relative to the slice spacing, which can be found in the image metadata. For example, if the slice spacing was $3 \mathrm{~mm}$, the resultant curve is shrunk inward $3 \mathrm{~mm}$. Also the 
contour center of segment organ in the current slice is used as the center for propagated contour curve.

\section{EXPERIMENTAL RESULTS}

The proposed method was applied to three abdominal CT volume datasets. The first data set consists of 130 slices for the liver organ, with slice thickness of $2 \mathrm{~mm}$, the second data set consists of 28 slices for the liver organ, with slice thickness of $5 \mathrm{~mm}$, whereas the third data set contains 14 slices for the spleen organ, with slice thickness of $5 \mathrm{~mm}$. Since we are re-using an existing level-set based active contour approach [19], of course the accuracy of segmentation is limited by the choice of the underlying levelset approach.

For dataset $1 /$ liver, the initialization of the contour circle in the chosen slice (slice number 51, shown in Fig. 3), the segmentation result of liver in slice number 51 is in Fig. 4, the result of segmenting middle liver coronal view is shown in Fig. 7, the initialization of propagated contour curve in the previous slice (slice number 50, in Fig. 8.a). The initialization based on the segmentation result of slice number 51 and the contour curve shrunk inside the liver in slice number 50 , the segmentation result of slice number 50 in Fig. 8.b, the initialization of propagated contour curve in slice number 49 in Fig. 8.c, the segmentation result of slice number 49 in Fig. 8.d.

Fig. 9 shows the results of segmenting spleen in dataset 2. Initialization of the contour is in Fig. 9.a, the segmented result of the propagation of the contour initialization through the dataset and the results of segmentation on each slice are in Figs 9.b, 9.c and 9.d. However, the final results of 3D visualization of liver and spleen are illustrated in Fig. 10.a and Fig. 10.b respectively.

TABLE I. TIME MEASURMENTS OF MANUAL AND AutomatiC INTILIZATION OF CONTOUR CURVE

\begin{tabular}{|c|c|c|c|}
\hline \multirow{2}{*}{$\begin{array}{c}\text { Dataset/ } \\
\text { Organ }\end{array}$} & \multicolumn{2}{|c|}{ Initialization Method } & \multirow{2}{*}{$\begin{array}{c}\text { Time } \\
\text { Efficiency } \\
(\%)\end{array}$} \\
\hline & $\begin{array}{c}\text { Manual } \\
\text { time (s) }\end{array}$ & $\begin{array}{c}\text { Automatic } \\
\text { time (s) }\end{array}$ & \\
\hline \#1/Liver & 59318 & 19085 & 310.80 \\
\hline \#2/Liver & 4256 & 2458 & 173.15 \\
\hline \#3/Spleen & 488 & 390 & 125.13 \\
\hline Average & & & 203.03 \\
\hline
\end{tabular}

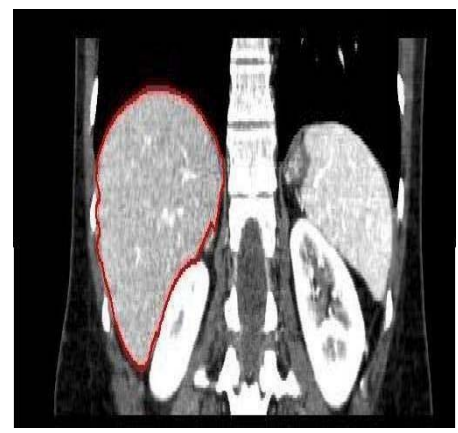

Figure 7. Segmented liver in coronal view.

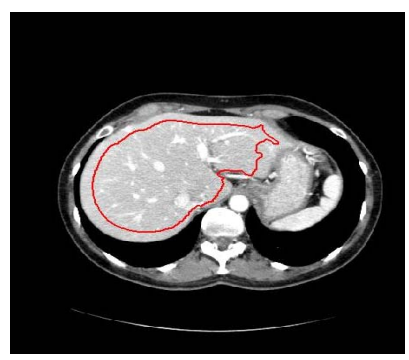

(a)

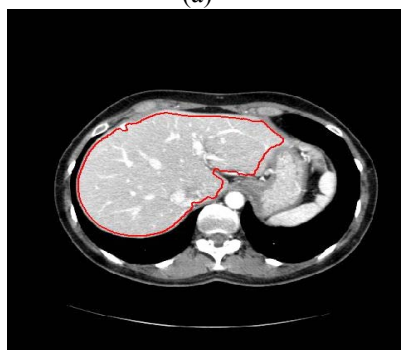

(c)

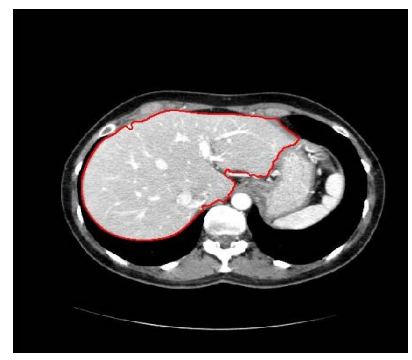

(b)

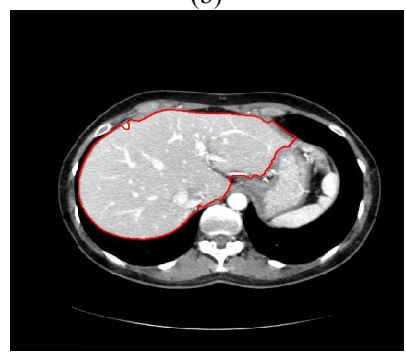

(d)
Figure 8. (a) Initial contour in liver slice 50; (b) Segmented liver slice 50; (c) Initial contour in liver slice 49; (d) Segmented liver slice 49.

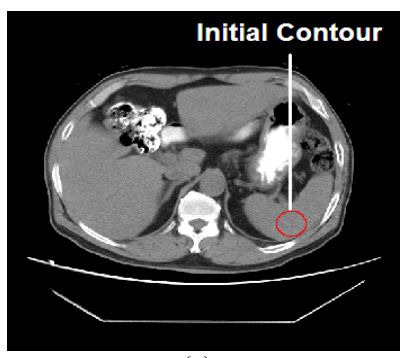

(a)

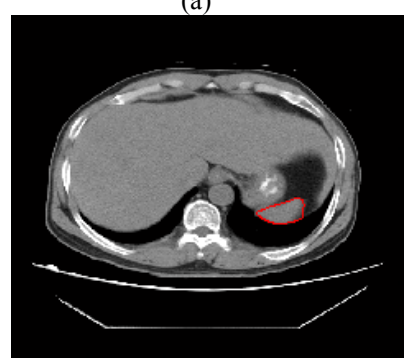

(c)

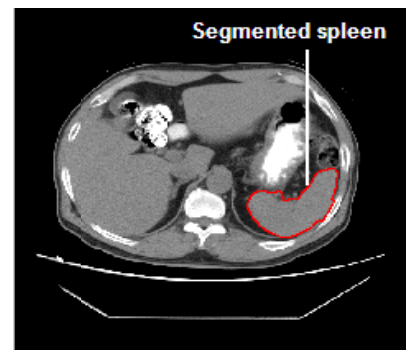

(b)

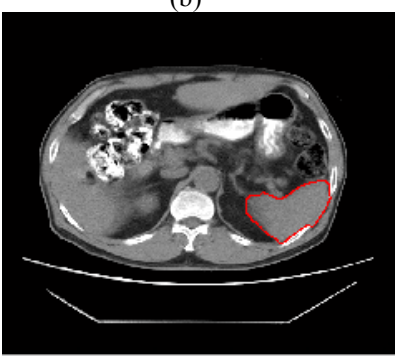

(d)
Figure 9. (a) Initial contour slice 9; (b) Segmented spleen slice 9; (c) Segmented spleen slice 2; (d) Segmented spleen slice 13.

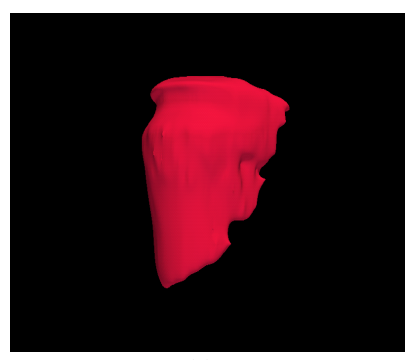

(a)

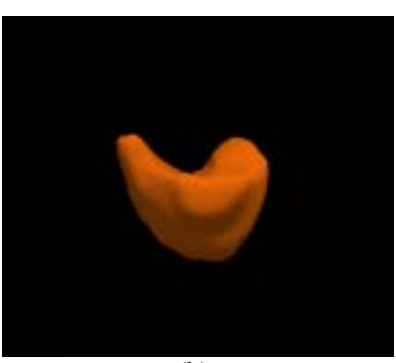

(b)
Figure 10. 3D visualization of segmented (a) liver and (b) spleen. 
For the efficiency of processing time, we calculate the time required to complete the level-set segmentation for all slices representing an organ in the three datasets. The time is calculated from the initialization of the contour curve in each slice until completing the segmentation for all slices. Table. 1 represents the time measurements that we got for both manual initialization of contour curve in each slice inside the dataset and automatic initialization which is our proposed technique. Time efficiency is calculated as in (8).

$$
\text { Time efficiency }=\left(\frac{\text { Time manual }}{\text { Time automatic }}\right) \times 100 \% \text {. }
$$

We notice that the time efficiency increase with the increase of the number of segmented slices. Dataset1/liver consists of 130 slices the efficiency is $310.80 \%$, dataset2/liver consists of 28 slices the efficiency is $173.15 \%$ and dataset $3 /$ spleen consists of 14 slices the efficiency is $125.13 \%$, while the average of efficiency is equal to $203.03 \%$.

\section{CONCLUSION}

In this paper we proposed a method to reduce the manual interaction in segmenting major anatomies in a CT volume dataset, using prior knowledge implemented in multiple views. Our method used level set based active contours and required multiple views of anatomy to be segmented. Several novel ideas were introduced in this paper. These are: using multiple views of abdominal dataset as prior knowledge to guide the initialization of level set contour, use of coronal view to reduce the number of processed axial slices and use of slice spacing from metadata to keep propagating the contour curve inside the target organ. This combination of ideas produced a technique which eliminates the need for manual initialization of contour curve in each slice in the dataset and greatly reduces the current time consuming and elaborate procedures of segmenting major organs in large volume datasets. It could be concluded based on the results of this study that the proposed method can handle the dissimilarity in intensity and shape between different datasets and produce better results in abdominal CT scans.

\section{ACKNOWLEDGMENT}

This research has been made possible through the Science Fund Grant Delineation and 3D Visualization of Tumor and Risk Structures" (DVTRS), No: $1001 /$ PKOMP/817001 by the Ministry of Science, Technology and Innovation of Malaysia.

\section{REFERENCES}

[1] L.R. Varshney, "Abdominal Organ Segmentation in CT Scan Images: A Survey,", Editor ed.^eds., 2002, pp.

[2] C. Elena, C. Paola, P. Stella, and L. Gabriele, "Automatic Abdominal Organ Segmentation from CT images," ELCVIA2009.

[3] 3. L. Chien-Cheng, and C. Pau-Choo, "Recognizing abdominal organs in CT images using contextual neural network and fuzzy rules," Proc. Engineering in Medicine and Biology Society, 2000.
Proceedings of the 22nd Annual International Conference of the IEEE, 2000, pp. 1745-1748 vol.1743.

[4] J.E. Koss, F.D. Newman, T.K. Johnson, and D.L. Kirch, "Abdominal organ segmentation using texture transforms and a Hopfield neural network," Medical Imaging, IEEE Transactions on1999, pp. 640-648.

[5] F. Ding, W. Leow, and S.-C. Wang, "Segmentation of 3D CT Volume Images Using a Single 2D Atlas," Computer Vision for Biomedical Image Applications, 2005, pp. 459-468.

[6] P. Hyunjin, P.H. Bland, and C.R. Meyer, "Construction of an abdominal probabilistic atlas and its application in segmentation," Medical Imaging, IEEE Transactions on2003, pp. 483-492.

[7] S. Pan, and B.M. Dawant, "Automatic 3D segmentation of the liver from abdominal CT images: a level-set approach," Proc. Medical Imaging 2001: Image Processing, SPIE, 2001, pp. 128-138.

[8] J. Lee, N. Kim, H. Lee, J.B. Seo, H.J. Won, Y.M. Shin, and Y.G. Shin, "Efficient Liver Segmentation exploiting Level-Set Speed Images with 2.5D Shape Propagation," 3D segmentation in the clinic: A grand Challenge2007, pp. 189-196.

[9] J. Garamendi, N. Malpica, J. Martel, and E. Schiavi, "Automatic Segmentation of the Liver in CT Using Level Sets Without Edges," Pattern Recognition and Image Analysis, 2007, pp. 161-168.

[10] A. Jamal, C, H. line, F. Geoffroy, B. Isabelle, and A. Elsa, "From generic knowledge to specific reasoning for medical image interpretation using graph based representations," Book From generic knowledge to specific reasoning for medical image interpretation using graph based representations, Series From generic knowledge to specific reasoning for medical image interpretation using graph based representations, ed., Editor ed.^eds., Morgan Kaufmann Publishers Inc., 2007, pp.

[11] A. Pitiot, H. Delingette, N. Ayache, and P.M. Thompson, "Expert Knowledge Guided Segmentation System for Brain MRI," Medical Image Computing and Computer-Assisted Intervention - MICCAI 2003, 2003, pp. 644-652.

[12] A.H. Zhuang, D.J. Valentino, and A.W. Toga, "Skull-stripping magnetic resonance brain images using a model-based level set," NeuroImage2006, pp. 79-92.

[13] T. de Araujo Buck, H.-H. Ehricke, W. Strasser, and L. Thurfjell, "3-D segmentation of medical structures by integration of raycasting with anatomic knowledge," Computers \& Graphics, 1995/6// 1995, pp. 441-449.

[14] A. Shimizu, R. Ohno, T. Ikegami, H. Kobatake, S. Nawano, and D. Smutek, "Segmentation of multiple organs in non-contrast 3D abdominal CT images," International Journal of Computer Assisted Radiology and Surgery2007, pp. 135-142.

[15] G. Srinivasa, V.S. Oak, S.J. Garg, M.C. Fickus, and J. Kovacevic, "Voting-based active contour segmentation of fMRI images of the brain," Proc. Image Processing, 2008. ICIP 2008. 15th IEEE International Conference on, 2008, pp. 1100-1103.

[16] O. Stanley, and A.S. James, "Fronts propagating with curvaturedependent speed: algorithms based on Hamilton-Jacobi formulations," J. Comput. Phys.1988, pp. 12-49.

[17] R. Malladi, J.A. Sethian, and B.C. Vemuri, "Shape Modeling with Front Propagation: A Level Set Approach," IEEE Transactions on Pattern Analysis and Machine Intelligence1995, pp. 158-175.

[18] T.F. Chan, and L.A. Vese, "Active contours without edges," Image Processing, IEEE Transactions on2001, pp. 266-277.

[19] L. Chunming, X. Chenyang, G. Changfeng, and M.D. Fox, "Level set evolution without re-initialization: a new variational formulation," Proc. Computer Vision and Pattern Recognition, 2005. CVPR 2005. IEEE Computer Society Conference on, 2005, pp. 430-436 vol. 431.

[20] V. Caselles, F. Catté, T. Coll, and F. Dibos, "A geometric model for active contours in image processing," Numerische Mathematik1993, pp. 1-31 